\title{
La competitividad en una empresa forestal comunitaria
}

\section{Competitiveness in a community forestry company}

Victor Hernández-Bautista'; Danae-Duana Ávila²; Zenón-Santiago López³

Artículo de investigación:

Fecha de recepción:

2019/09/22

Fecha de aceptación:

2019/12/16

Esta publicación se encuentra bajo licencia:

Creative Commons ReconocimientoNoComercialSinObraDerivada 4.0 Internacional

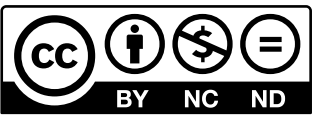

\section{Resumen}

El aprovechamiento forestal desempeña un papel fundamental para el abastecimiento de materia prima a la industria forestal y al desarrollo socioeconómico de las comunidades forestales, dado su potencial natural en proporcionar bienes y servicios. Existen diversos estudios del manejo forestal, pero, pocos sobre competitividad de las cadenas productivas. La presente investigación se llevó a cabo en la comunidad forestal de Capulalpam de Méndez, Ixtlán, Oaxaca, México y se considera un diseño no experimental debido a que se observó el fenómeno tal y como se da en su contexto natural, para posteriormente analizarlo sin manipular variables. Los resultados obtenidos muestran que los indicadores: Productividad, calidad del producto, rentabilidad y participación en el mercado, con valores de $0.75,0.75,0.70$ y 0.62 respectivamente, son los indicadores más importantes que determinan el logro de la competitividad de la empresa.

Palabras clave: Aprovechamiento forestal, competitividad, productividad, rentabilidad.

Doctor en Economía, Instituto de Estudios Ambientales Universidad de la Sierra Juárez, Oaxaca, México. victor1@unsij.edu.mx código ORCID: https:// orcid.org/0000-0002-3578-0129

2 Dr. En Economía, Universidad Autónoma de Estado de Hidalgo UAEH, Pe Administración, México. duana@uaeh.edu.mx, código ORCID: https://orcid. org/0000-0003-2286-2843

3 Ingeniero forestal, Secretaria de Seguridad Pública Unidad Ambiental, Morelia Michoacán. México. santiglopz@hotmail.com, código ORCID: https:// orcid.org/0000-0002-3534-7114 


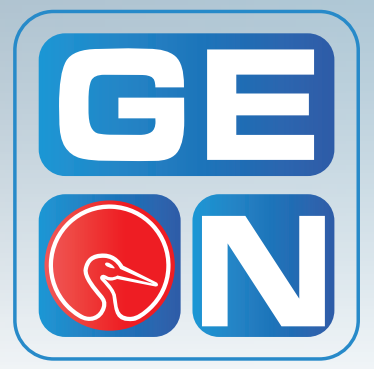

Revista GEON

(Gestión, Organizaciones y Negocios)

ISSN: 2346-3910 en línea

revistageon@unillanos.edu.co

Universidad de los Llanos

Colombia

Hernandez Bautista, V., Duana Avila, D., \& Santiago López, Z. (2020).

La competitividad en una empresa forestal comunitaria

Revista GEON

(Gestión, Organizaciones y Negocios), 7(1), 70-82.

\section{다켝}

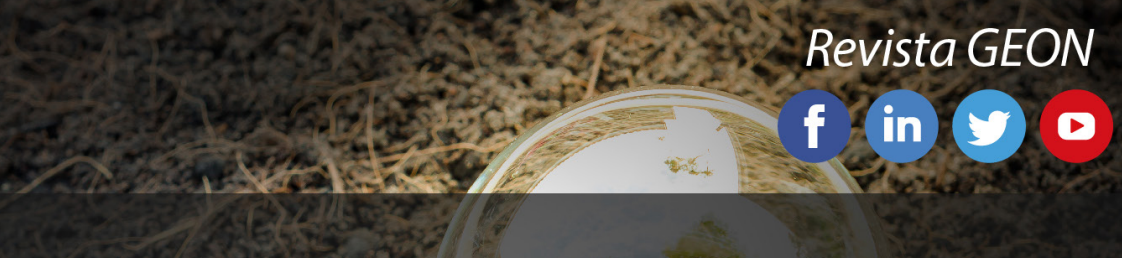

66 Los principales problemas que enfrenta la cadena productiva forestal se presenta en el proceso productivo, sobre todo, en la tecnología que resulta ser costoso y que imposibilita escalar en la cadena, así como las variables mercadotecnia y comercialización 99

Hernandez Bautista, V., Duana Avila, D, \& Santiago López, Z. (2020). La competitividad en una empresa forestal comunitaria. Revista GEON (Gestión, Organizaciones Y Negocios), 7(1), 70-82. http://revistageon.unillanos.edu.co 
Cómo citar este artículo Toreference this article:

Hernandez Bautista, V., Duana Avila, D., \& Santiago López, Z. (2020). La competitividad en una empresa forestal comunitaria. Revisto GEON (Gestión, Organizaciones Y Negocios), 7(1), 70-82. https://doi org/10.22579/23463910.179

\section{Abstract}

Forest utilization plays a fundamental role for the supply of raw material to the forest industry and the socio-economic development of forest communities, given its natural potential in providing goods and services. There are several studies of forest management, but few on competitiveness of productive chains. The present investigation was carried out in the forest community of Capulalpam de Méndez, Ixtlán, Oaxaca, Mexico and is considered a non-experimental design because the phenomenon was observed as it occurs in its natural context, to later analyze it without manipulating variables The results obtained show that the indicators: Productivity, product quality, profitability and market share, with values of $0.75,0.75,0.70$ and 0.62 respectively, are the most important indicators that determine the achievement of the company's competitiveness.

Keywords: Forest use, competitiveness, productivity, profitability.

\section{Introducción}

La ubicación geográfica de México, su topografía y climas, son factores esenciales para la existencia de la diversidad de ecosistemas forestales; se posee una superficie de $55.3 \mathrm{mi}$ llones de hectáreas (has) de bosques, de las cuales en 21.6 millones de hectareas se realizan aprovechamiento de productos forestales maderables y no maderables (INEGI, 2005). El estado de Oaxaca cuenta con una superficie total de 6.3 millones de has, de las cuales 5.1 millones corresponden a terrenos forestales que en su mayoría es de propiedad colectiva bajo el régimen de ejido o comunidad agraria (SEMARNAT, 2004). Los bosques templados cubren 2.7 millones de has y presentan asociaciones forestales de distintos tipos: bosque mesófilo de montaña, bosques de pino, bosques de pino-encino, bosques de encino-pino, bosques de encino y matorral de coníferas (Toledo \& Ordóñez, 1993).

A partir de 1982, la política de promoción de la silvicultura campesina tuvo expresiones particulares en distintos estados y en particular en Oaxaca. Varias comunidades formaron sus propias organizaciones para asumir el aprovechamiento forestal, como es el caso de Capulálpam de Méndez a través la conformación de la Unidad Económica Especializada de Aprovechamiento Forestal Comunal (U.E.E.A.F.C.) "San Mateo" de carácter 
comunal, la cual fue constituida el 1 de abril de 1984, y está integrada a la Unión de Comunidades Productoras Forestales Zapotecas-Chinantecas (UZACHI). La actividad principal de esta organización es el aprovechamiento forestal maderable bajo un programa de manejo validado por la Secretaría de Medio Ambiente y Recursos Naturales (SEMARNAT). La empresa comunal cuenta con equipo para la producción y transformación de madera en rollo, que le ha permitido ofrecer productos de buena calidad, aunque se han identificado algunas debilidades en cada una de las etapas de la cadena de valor, como lo es el desconocimiento de las etapas que generan mayores costos; esta problemática restringe la competitividad en cuanto a la venta de los recursos maderables a un mejor precio.

Uno de los recursos naturales de gran importancia a nivel social, económico y ecológico lo integra el recurso forestal del cual se obtienen bienes y servicios ambientales. Estos recursos satisfacen necesidades de consumo de diferente índole y el valor económico que tienen para la sociedad, a partir de los cuales se obtienen materias primas para otras industrias que generan bienes de consumo final, esto justifica la pertinencia de estudiar la eficiencia de los indicadores de competitividad para generar información que permita la planeación y la toma de mejores decisiones en el eslabón de aprovechamiento forestal.

Con base a lo anterior, el objetivo de esta investigación es evaluar los indicadores de que inciden en la competitividad de la empresa forestal comunitaria de Capulálpam de Méndez.

\section{Contexto teórico}

La capacidad de competir se relaciona con las ventajas que tienen los agentes que están compitiendo. Se señalan los conceptos de ventaja absoluta, ventaja comparativa y ventaja competitiva.

En un estudio realizado por la Comisión de Competitividad Industrial de los Estados Unidos en 1983, señala que la competitividad debe ser entendida como "el grado en que una nación puede, bajo condiciones de libre mercado, producir bienes y servicios que satisfagan los requerimientos de los mercados internacionales y, simultáneamente, mantener o expandir los ingresos reales de sus habitantes" (Citado por Cabrera M. A. et al., 2011).

\section{Ventaja competitiva}

Krugman (1990), considera que un análisis de la competitividad de una nación debería considerar los diversos determinantes del nivel de vida de la población, tales como el crecimiento, el empleo y la distribución de ingresos.

Sin duda alguna, Michael Porter, puede considerarse el más destacado portavoz del concepto de ventaja competitiva. Su preocupación fundamental es elaborar para las empresas el concepto microeconómico de competitividad. En su libro La Ventaja Competitiva de Las Naciones (1993), plantea que la estrategia competitiva establece el éxito o fracaso de las empresas. La estrategia se refiere a una habilidad o destreza, y la competitividad (es decir, la capacidad de hacer uso de esa destreza para permanecer 
en un ambiente), es entonces un indicador que mide la capacidad de una empresa de competir frente al mercado y con sus rivales comerciales. La competitividad es también la búsqueda de una posición relativamente favorable en un mercado, con respecto a los competidores, la cual le permitirá permanecer y expandirse.

Porter considera que no todas las actividades generan la misma rentabilidad, y que ésta debe ser suficiente, creciente y sostenida en el tiempo, con el fin de garantizar los elementos esenciales para la existencia de una empresa. Así, la competitividad es el grado en que un país, estado, región o empresa, produce bienes o servicios bajo condiciones de libre mercado, enfrentando la competencia de los mercados nacionales o internacionales, mejorando simultáneamente los ingresos reales de sus empleados y consecuentemente la productividad de sus empresas.

Porter (1993), plantea que las empresas competitivas hacen regiones (ciudades y territorios) competitivas y, por lo tanto, naciones poseedoras de esta cualidad; asimismo, supone que la sumatoria de empresas competitivas, es decir, generadoras de rentabilidad en estas condiciones, dará como resultado países con mayor riqueza para sus habitantes y mayor bienestar general.

\section{Estrategia competitiva}

La unidad básica del análisis para comprender la competencia es el sector. Un sector es un grupo de competidores que fabrican productos o prestan servicios y compiten direc- tamente unos con otros. Un sector estratégicamente diferenciado comprende productos en que las fuentes de ventaja competitiva son similares (Porter, 1991).

La estrategia competitiva consiste en ser diferente; significa elegir deliberadamente un conjunto de actividades diferentes para prestar una combinación única de valor, es decir, la esencia de la estrategia está en las actividades, en la decisión de realizarlas de manera diferente que los rivales (Porter, 2006). Para alcanzar una posición en el sector, Porter releva tres estrategias genéricas para lograr esa posición.

La primera, es la estrategia de liderazgo en costos y consiste en que la organización o empresa se propone convertirse en el fabricante de costo más bajo de su industria. La segunda, es la estrategia de diferenciación, a través de esta la empresa intenta distinguirse dentro de su sector en algunos aspectos ampliamente apreciados por los compradores. Escoge uno o más atributos importantes y adopta un posicionamiento especial para atender esas necesidades y obtiene un precio alto de sus productos. La tercera, la estrategia de concentración la cual se basa en la elección de un estrecho ámbito competitivo dentro de un sector industrial. La empresa selecciona un segmento o grupo de segmentos del sector y adapta su estrategia para atenderlos excluyendo los restantes (Porter, 2006).

\section{Competitividad empresarial}

En cuanto al concepto de competitividad empresarial, se puede plantear 
que, al igual que la competitividad en general, éste presenta un sin número de definiciones y no es fácil encontrar una en la que todos estén de acuerdo. Sin embargo, se puede concluir a partir de la revisión de diferentes acercamientos que los elementos comunes son: además de la participación en el mercado, la productividad, la alta calidad de los productos ofrecidos y los bajos costos; que incluye también el criterio de la innovación. Además de Porter (1993), que insiste en la importancia del proceso productivo como criterio determinante de la competitividad; por su parte, Alic (1987) la define como "la capacidad de las empresas para diseñar, desarrollar, producir y colocar sus productos en el mercado internacional en medio de la competencia con empresas de otros países".

Reinel \& Bermeo (2005), afirma que la competitividad de la empresa es el rendimiento valorado por la capacidad que tiene para generar más valor agregado que sus competidores. Otros autores; como Vallejo (1999); Porter (1981) \& Valero (2004), insisten en la permanencia de las empresas en el mercado libre como un criterio de competitividad. La importancia de la innovación en la generación de productos es destacada por Román (2004) \& Pallares (2004) da gran importancia tanto a la capacidad de generar redes como a la asociación.

Malaver (1999), señala que la competitividad es "la necesidad de las organizaciones de sostenerse y consolidarse dentro de sus mercados, teniendo como indicador el porcentaje de participación de sus bienes y servicios en ellos, a partir de una concepción sistemática que incorpora elementos económicos, empresariales, políticos y socioculturales".

Para Ruesga \& Da Silva (2007), la competitividad a nivel de empresas se define como la capacidad de las mismas para competir en los mercados, ganar mercado, aumentar sus beneficios y crecer; en este sentido, los indicadores de competitividad más utilizados son: la cuota de mercado, los beneficios, los dividendos y el volumen de inversiones. Los principales factores determinantes de la evolución de la competitividad son: por un lado, los precios y los costos de los insumos (el factor trabajo sigue siendo de los más importantes) y por otro, los factores tecnológicos y organizativos (Torres-Flórez, 2016).

Entre estos últimos destacan; calidad de los productos, economías de escala, tecnología de proceso y de producto, organización de la producción, sistema de distribución eficiente, asistencia postventa adecuada y capacidad motivacional de los trabajadores. La utilización o desarrollo relativamente más eficiente de estos factores afectan positivamente a los indicadores de competitividad, proporcionando mayor nivel competitivo a las empresas (Ruesga \& Da Silva, 2007).

\section{Materiales y Métodos}

La comunidad de Capulálpam de Méndez se localiza en la región Sierra Norte en las coordenadas 17020'53" y $17^{\circ} 20^{\prime} 05^{\prime \prime}$ latitud norte y $96^{\circ} 24^{\prime} 09^{\prime \prime}$ y $96^{\circ} 23^{\prime 2} 24^{\prime \prime}$ longitud oeste, al noreste 
de la ciudad de Oaxaca. Con altitudes que varían desde 2000 a 3000 msnm (INEGI, 1984). Cuenta con una superficie de 3,850 hectáreas, limita al norte con los terrenos comunales de Ixtlán de Juárez y San Juan Tepanzacoalcos, al sur con terrenos comunales de San Juan Juquila Vijanos, Santiago Xiacui y pueblos mancomunados (Lachatao, Amatlán y Yavesía); al este con la comunidad de San Miguel Yotao y al oeste con terrenos comunales de Santa María Yahuiche (ver Figura 1).

\section{Características físicas ambientales}

\section{Suelo}

Los suelos predominantes en el área de estudio la FAO/UNESCO lo clasificó como litosoles, rendzinas y cambiso- les de textura limosa. En la zona de bosque de coníferas los suelos son delgados y frecuentemente contienen únicamente una capa formada por materia orgánica proveniente de la caída de las hojas de pino y encino, su reacción es de ácida a muy ácida (rangos de pH que van de 2.8 a 5), con alta capacidad de saturación de bases (Castaños, 1962; FAO-UNESCO, 1990). El suelo de clasificación litosol es de color negro o gris muy oscuro, con altos contenidos de nutrientes, profundidad desde $10 \mathrm{~cm}$ en laderas y pendientes abruptas con alto riesgo de erosión, hasta $50 \mathrm{~cm}$ de profundidad en los valles (FAO/UNESCO, 1990).

El suelo de clasificación cambisol presenta horizontes bien definidos cuyo horizonte B es argílico, se localiza en mayor proporción en áreas de lome-

Figura 1. Localización de la Comunidad de Capulálpam de Méndez, Oaxaca

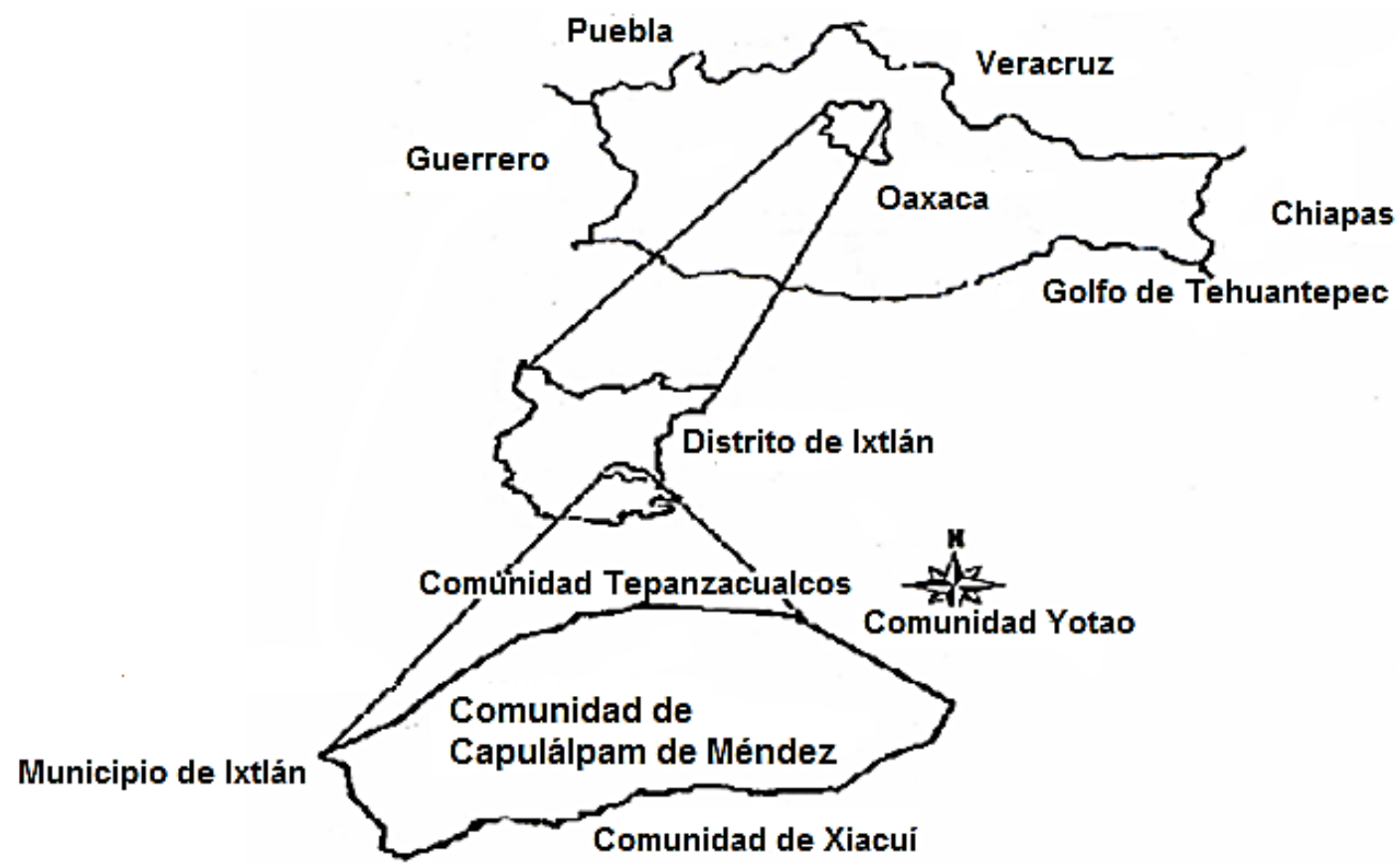

Fuente: UZACHI, (2003a) 
ríos, sierras y montañas del lugar, con pequeña proporción se les localiza en declives suaves y laderas. Generalmente es de coloración parda a rojizo de textura migajón arcillosa, reacción del suelo ligeramente ácida con procesos de desbasificación (FAO/UNESCO, 1990).

\section{Clima}

La temperatura promedio anual es de $15.2{ }^{\circ} \mathrm{C}$. La precipitación total es de 1,115 mm anuales y los meses con mayor intensidad de lluvias se presentan en los meses de junio hasta octubre. El clima de la comunidad según la clasificación de Koppen modificado por García (1987), corresponde a C (w2) (w) big: templado sub-húmedo, con abundantes Iluvias en verano, se caracteriza además por presentar entre 30 y 59 días, de 150 a 200 mm de lluvia invernal, por lo que se puede considerar con sequía ligera, lo cual permite un buen desarrollo de la vegetación. Durante el invierno se acentúa el frío en los meses de diciembre hasta febrero. En la estación de la primavera se registra una época de calor seco, principalmente en los meses de marzo, abril y mayo (Castaños, 1962; García, 1987; INEGI, 1993).

\section{Tipo de vegetación}

De acuerdo con Rzedowski (1978), en el área de estudio se presentan bosques de pino-encino y encino. El relieve es montañoso y la gran amplitud altitudinal que va desde 2000 hasta 3000 msnm, así como la exposición predominante suroeste da lugar a una marcada variación de condicio- nes climáticas que a su vez influyen en la diversidad de tipos de vegetación. A esto se suma la variación en las condiciones edáficas y la influencia humana, para dar lugar a una gran heterogeneidad de la cubierta vegetal.

El bosque de pino-encino está conformado por las especies: Pinus patula, P. pseudostrobus, P. ayacahuite, con Quercus laurina, Quercus spp., Abies sp., Arbutus xalapensis, Alnus y Buddleia. Ocasionalmente se encuentran rodales pequeños con Abies hickeli, Cupressus lusitánica y Taxus globosa (Miranda \& Hernández, 1963; UZACHI, 1993). En los bosques de pino-encino y encino se pueden encontrar también especies de menor importancia económica pero que tienen importancia ecológica tales como son Ternstroemia sp, Persea sp, Litsea glauscescens, Fraxinus sp, Oreopanax xalapensis, Rapanea jurgensenii y Symplocos sp. (Salas, 2002).

\section{Diseño de la investigación}

El propósito del diseño de la investigación es responder a las preguntas de investigación, cumplir con los objetivos del estudio. La presente investigación se considera un diseño no experimental debido a que se observó el fenómeno tal y como se da en su contexto natural, para posteriormente analizarlo sin manipular variables. El tipo de diseño no experimental que se utilizó en esta investigación es de carácter transeccional o transversal, es decir, los datos se obtuvieron en un solo momento, donde las variables se analizaron en un momento dado (Hernández, 2010). 


\section{Unidad de análisis y tamaño de muestra}

El presente estudio ha considerado como unidad de análisis a las personas involucradas en el eslabón de aprovechamiento forestal de la cadena productiva (Servicios técnicos, Comisariado de bienes comunales, empresa dedicada a la extracción de la madera (contratista), personal administrativo y operativo de la Unidad Económica Especializada de Aprovechamiento Forestal Comunal (U.E.E.A.F.C.) "San Mateo"; de la comunidad de Capulálpam de Méndez, Oaxaca.

De esta unidad de análisis se tomó una muestra "no probabilística" conocida también como "dirigida", debido a las posibilidades tenidas para entrevistar a la totalidad de la población escogida, de esta forma se consideraron (integrantes del comisariado de bienes comunales, al director técnico de UZACHI.

\section{Operacionalización de variables}

La variable que comprende esta investigación es competitividad a continuación, se operacionalizan las variables correspondientes como se observa en el Cuadro 1 en el caso del eslabón de aprovechamiento forestal.

\section{Instrumentos de recolección de datos}

Para realizar la investigación y recolectar la información, se utilizó la técnica de la encuesta, por lo que se elaboró un cuestionario como herramienta guía para plantear las preguntas y codificar las respuestas de los actores involucrados, en cada eslabón de la cadena productiva forestal de Capulálpam de Méndez, Oaxaca.

El cuestionario desarrollado tiene como base preguntas de tipo cerrada, es decir, contiene categorías u opciones de respuesta que han sido previamente delimitadas.

La escala empleada en las preguntas son de tipo Likert, es decir, se trata de un conjunto de ítems presentados en forma de afirmación o juicios; ante los cuales se pide la respuesta de los actores involucrados en el eslabón de aprovechamiento forestal

Cuadro 1. Operacionalización de la variable competitividad eslabón aprovechamiento forestal

\begin{tabular}{lll} 
Variable & \multicolumn{1}{c}{ Índice } & Ítems \\
& Rentabilidad & 49,50 \\
& Permanencia en el mercado & 51,53 \\
& Productividad & 54,55 \\
Competitividad & Participación en el mercado & 56,60 \\
& Prestigio & 61,62 \\
& Lealtad del cliente & 63 \\
& Costos & 64,65 \\
& Calidad del Producto & 66 \\
\hline
\end{tabular}

Fuente: Elaboración propia, con información de Porter, (2007). 
de la cadena productiva forestal de la comunidad. En algunas ocasiones se emplean preguntas de diferencial semántico en función de la escala Likert (ver cuadro 2).

\section{Logística de campo}

1. Prueba piloto: Consistió en administrar el instrumento de recolección de datos (cuestionario) a una pequeña parte de la población total con el objeto de probar la pertinencia y eficacia de las preguntas, así como el comportamiento de las variables e indicadores para su corrección y mejora.

2. Versión final: De acuerdo a las observaciones y debilidades encontradas en la implementación y diseño del cuestionario piloto, se hicieron cambios para adecuar correctamente el instrumento de medición, de esta forma, se redactó el cuestionario final.

3. Aplicación de cuestionario: Se aplicó a los actores involucrados del eslabón de aprovechamiento forestal.

4. Base de datos: Comprendió la construcción de una base de datos en el programa Microsoft Office Excel 2013, para la construcción de índices, e indicadores, una vez teniendo la base de datos se procedió a establecer una escala de 0 a 1, y para ello se estandarizaron los datos mediante la fórmula (Arroyo \& Púa, 2011).

Dónde:

$\mathbf{Z i}=$ Puntaje estandarizado variable i.

$\mathbf{X} \mathbf{i}=$ Valor que toma la variable i.

min= Valor mínimo variable i.

max= valor máximo variable i.

5. Resultados: Por último, se realizó el análisis descriptivo de los resultados, así como, las correlaciones entre las variables, comentarios y sugerencias del trabajo.

\section{Análisis y discusión.}

Para la variable competitividad y sus indicadores los cuales se componen de: rentabilidad, permanencia en el mercado, productividad, participación en el mercado, prestigio, lealtad del cliente, costos y calidad del producto. Los indicadores con valores más al-

Cuadro 2. Opciones de la escala Likert empleadas

\begin{tabular}{ccccc}
\hline Nunca & $\begin{array}{c}\text { La mayoría de las } \\
\text { veces no }\end{array}$ & $\begin{array}{c}\text { Algunas veces sí, } \\
\text { algunas veces no }\end{array}$ & $\begin{array}{c}\text { La mayoría de las } \\
\text { veces si }\end{array}$ & Siempre \\
\hline $\begin{array}{c}\text { Totalmente en } \\
\text { desacuerdo }\end{array}$ & En desacuerdo & $\begin{array}{c}\text { Ni de acuerdo ni } \\
\text { en desacuerdo }\end{array}$ & De acuerdo & $\begin{array}{c}\text { Totalmente de } \\
\text { acuerdo }\end{array}$ \\
\hline Pésimo o muy mal & Mal & Regular & Bien & $\begin{array}{c}\text { Muy bien o } \\
\text { excelente }\end{array}$ \\
Escala de diferencial semántico en función a la escala Likert & Begular & Barato & Muy barato \\
\hline
\end{tabular}

Fuente: Tomado de Hernández, (2010). 
tos son: Prestigio, lealtad del cliente y permanecía en el mercado; la comunidad de Capulálpam de Méndez es reconocido como productor de madera en la región como también es reconocido de producir madera de buena calidad, el principal cliente demandante de la madera en rollo es la U.E.E.A.F.C. "San Mateo" perteneciente a la misma comunidad, la cual por políticas de la comunidad está obligada a comprar la materia prima por ya más de 20 años. El prestador de servicios técnicos ha tenido prestigio en la región por sus servicios, de igual forma la comunidad como cliente de los servicios técnicos le ha sido leal por el buen servicio que recibe.

En cuando a los indicadores: Productividad, calidad del producto, rentabilidad y participación en el mercado, con valores de $0.75,0.75,0.7$ y 0.62 individualmente, son los indicadores más importantes para poder lograr la competitividad. Los actores involucrados en el eslabón de aprovechamiento forestal están de acuerdo que la tecnología y la capacitación contribuye aumentar la productividad y la calidad de la madera en rollo. Sus ganancias son regulares y la mayor parte es reinvertido al bosque; en reforestaciones, brechas corta fuego, vigilancias y en adquisición de equipos. En cuanto a participación en el mercado consideran no tener problemas por que el 90\% de su madera en rollo es vendida en el mercado local y solo el $10 \%$ se vende al mercado de la ciudad de Oaxaca y principalmente es madera de corta dimensión (bolo) y el prestador de servicios técnicos ofrece el $80 \%$ de sus servicios a las comunidades de la sierra Juárez y estas son las que integran a la UZACHI, El 20\% de sus servicios ofrece a otras comunidades forestales.

En la extracción de la madera en rollo se debe poner especial atención al indicador de Costos, ya que los insumos utilizados registran altos precios que repercuten en los costos de producción, misma que incide en menor competitividad (ver Figura 2).

Figura 2. Valores de los indicadores de la competitividad

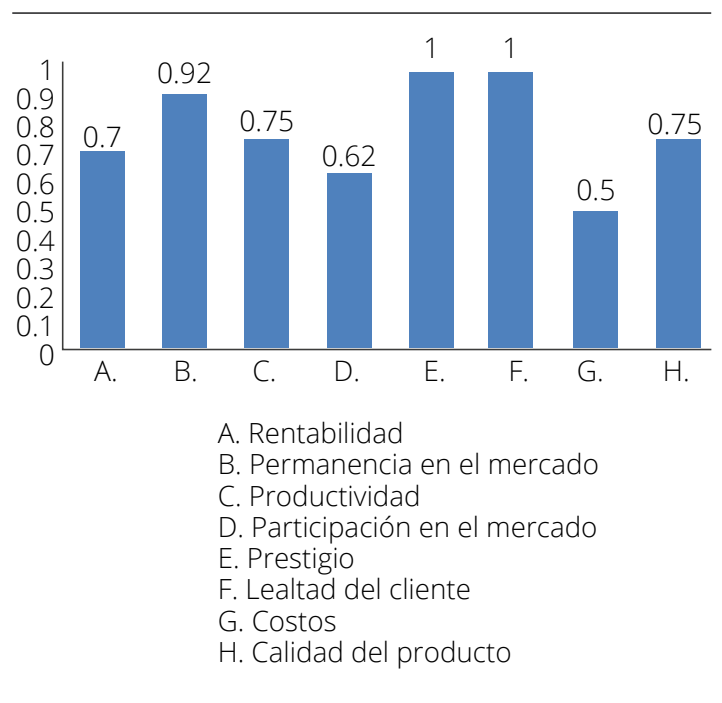

Fuente: Elaboración propia con datos de encuestas.

\section{Conclusiones}

A continuación, se presentan las conclusiones del estudio, con énfasis de los resultados sobre el sistema de valor del aprovechamiento forestal en la comunidad de Capulálpam de Méndez, Oaxaca; además de los datos estadísticos descriptivos obtenidos a partir del cuestionario. El análisis del sistema de valor nos permite obtener los cuellos de botella, los puntos críticos y las ventajas competitivas para mejorar en cada uno de los indicadores de competitividad de la cadena de 
producción forestal. En el sistema de valor de la madera existen intermediarios que restan valor a la producción forestal y reducen los márgenes de comercialización para el productor, ofreciendo precios bajos del valor real del producto. La U.E.E.A.F.C. "San Mateo" ha logrado tener vínculos directos con eslabón de transformación tanto de madera en rollo como de madera aserrada lo que le da posibilidad de escalar a los tres eslabones de la cadena productiva y expandirse a otros mercados.

Los principales problemas que enfrenta la cadena productiva forestal se presenta en el proceso productivo, sobre todo, en la tecnología que resulta ser costoso y que imposibilita escalar en la cadena, así como las variables mercadotecnia y comercialización que no han sido desarrolladas.

La ventaja competitiva de la U.E.E.A.F.C. "San Mateo" se centra en el manejo sustentable de los recursos naturales que les proporcionan mayores beneficios sociales, económicos y ambientales de manera directa e indirecta, sin embargo, su estructura organizacional y visión empresarial limita el acceso a los financiamientos que otorgan los distintos programas gubernamentales.

\section{Referencias}

Cabrera M.A.M, P.A López L. \& M.C. Ramírez. (2011), La competitividad Empresarial: Un marco conceptual para su estudio, documento de investigación, Universidad Central de Colombia, Bogotá D.C. Colombia.
Castaños, L. (1962). Evaluación de la calidad de estación de Pinus patula en el norte de Oaxaca. Boletín Técnico. No.2. INIF. México. 32 p

FAO-UNESCO. (1990). Mapa mundial de suelos. Informes sobre recursos mundiales de suelos. Organización de las Naciones Unidas para la Agricultura y la Alimentación. Roma. 60: 142 p.

García., E. (1987). Modificaciones al sistema de clasificación climática de Köppen. Instituto de Geografía, UNAM. México. 246 p.

INEGI (Instituto Nacional de Estadística, Geografía e Informática (INEGI).

INEGI (Instituto Nacional de Estadística, Geografía e Informática (INEGI). (1984). Carta topográfica. E14-D28 Esc. 1: 50, 000 Oaxaca, Oax., México. (2005). Superficie forestal por ecosistema según formación primaria y vegetación secundaria en el año 2000. Boletín informativo. Sistemas Nacionales Estadístico y de Información Geográfica (SNEIG). 33 p.

Krugman, P. (1990). Rethinking International Trade. MIT Presss, Cambridge, MA.

Malaver, F. (1999). Lecturas sobre competitividad, empresa y educación gerencial. Bogotá: Centro Editorial Javeriano.

Miranda, F. \& X. Hernández. (1963). Los tipos de vegetación de México y su clasificación, Bol. Soc. Bot. Méx., Num. 28: 28-79. México, D. F.

Pallares, Z. (2004). La asociatividad empresarial como estrategia para la competitividad empresarial. En: Valencia, R. y Muñoz, J (comps.) Asociatividad empresarial en Colombia, 57-63. Bogotá: CAF Mincomercio, Cámara de Comercio de Bogotá - Acopi - ANDI - IICA. 
Porter, M (1987). From Competitive Advantage to Corporate Strategy. Harvard Business Review, May 1987.

Porter, M (1991). La Ventaja Competitiva de las Naciones. (Buenos Aires: Editorial Vergara, p.125

Porter, M (1993). La ventaja competitiva de las naciones. Buenos Aires: Ediciones Javier Vergara.

Porter, M (2006). Ventaja competitiva. Quinta reimpresión. México: CECSA.

Reinel, J. \& E. Bermeo. (2005). Las directrices del costo como fuentes de ventajas competitivas. Revista Estudios Gerenciales. 81-103

Román, R. (2004). Teorías y mediciones de competitividad: una perspectiva. Bogotá: Universidad Distrital Editores.

Ruesga, M. \& J. Da Silva. (2007). Competitividad y globalización: Nuevos y viejos desafíos. Papeles del Este, número 14, $1-27$.

Rzedowski, J. (1978). Vegetación de México. Editorial Limusa. México D.F. 432 p

Salas, S. (2002). Relación entre la heterogeneidad ambiental y la variabilidad estructural de los bosques de pino encino de Oaxaca, México. Tesis de maestría de la Facultad de Ciencias de la Universidad Nacional Autónoma de México, D.F. 101 p.
SEMARNAT. (2004). Documento estratégico rector del inventario forestal nacional y de suelos. Manual Técnico Operativo de la SEMARNAT. 22(2):22-79.

Toledo, V. \& M. A. Ordoñez De J. (1993). The Biodiversity Scenario of Mexico: A Revies of Terrestrial Habitats, En: T.P. Ramamoorthy, R. Bye, A. Lot y J. Fa (eds.) Biological Diversity of Mexico: Origins and Distributions. Oxford University Press, New York. Pp. 757-777.

Torres-Flórez, D. (2016). El liderazgo adecuado para dirigir los aspectos ambientales en una organización. Revista GEON (Gestión, Organizaciones Y Negocios), 3(2), 5. https://doi. org/10.22579/23463910.54

UZACHI. (1993). Dirección Técnica Forestal. Programa de Manejo Forestal para el predio de Capulálpam de Méndez, Municipio del mismo nombre. Distrito de Ixtlán, Oaxaca, México. 200 p.

Valero, M. (2004). Análisis de competitividad al sector del dulce en el área metropolitana de Bucaramanga. Bogotá: Ascolfa.

Vallejo, C. (1999). Competitividad: aproximaciones conceptuales. Bogotá: Centro de Estudios Regionales, Cafeteros y Empresariales (Crece). 